\title{
Diffusion and convection in nature
}

\author{
Alberto Vailati ${ }^{1}$ (D), Shenghua $\mathrm{Xu}^{2}$, Stefano Aime $^{3}$ (D), and Fabrizio Croccolo ${ }^{4, a}$ (D) \\ 1 Dipartimento di Fisica 'Aldo Pontremoli', Università degli Studi di Milano, Milan, Italy \\ ${ }^{2}$ Key Laboratory of Microgravity, Institute of Mechanics, Chinese Academy of Sciences, Beijing, China \\ 3 Matière Molle et Chimie, Ecole Supérieure de Physique et Chimie Industrielles, Paris, France \\ ${ }^{4}$ E2S UPPA, CNRS, TotalEnergies, LFCR UMR5150, Universite de Pau et des Pays de l'Adour, Anglet, France \\ Published online 30 November 2021 \\ (C) The Author(s), under exclusive licence to EDP Sciences, SIF and Springer-Verlag GmbH Germany, \\ part of Springer Nature 2021
}

Abstract We present the Topical Issue 'Diffusion and Convection in Nature'.

\section{Introduction}

Diffusion and convection are the two facets of mass transport phenomena in fluids. While diffusion stems from the thermal motion of molecules at the microscale, convection involves the collective motion of particles at the macroscale. Both phenomena can be observed in many natural systems, even beyond the realm of fluid dynamics. Several studies have focused, for example, on the diffusion of languages in human communities [1], as well as on viruses, as in the case of the recent COVID19 pandemic [2]. Collective motion can be observed in the value of stocks as well as in the movement of flocks of birds [3], which are just a couple of examples of selforganised flow observed in systems other than fluids. In this Topical Issue, our specific aim was to attract contributions from authors working in more general fields of science, with the goal of sharing common knowledge on modelling tools, as well as statistical analysis of experimental data, or even numerical tools for simulating the similar behaviour of different systems.

\section{Topical issue}

The EPJE Topical Issue 'Diffusion and Convection in Nature' contains a total of seven scientific papers classified into two groups, namely 'diffusion' and 'convection'. The published papers are presented in scattered order in the following.

\subsection{Diffusion}

Diffusion is a fundamental transport process that stems from the movement of molecules due to thermal agitation at the microscale. It is driven by the presence of

\footnotetext{
a e-mail: fabrizio.croccolo@univ-pau.fr (corresponding author)
}

a gradient in one or more thermodynamic variables, and its effects can be observed at any scale, from the microscopic to the macroscopic. In recent years, much attention has been focused on the behaviour of non-equilibrium fluctuations and their long-range nature in the presence of a macroscopic gradient [4] that is the link between the random walk of molecules and the large-scale behaviour of diffusion in natural systems. In this Topical Issue, we host contributions in different fields where diffusion plays an important role. In the paper by Armin Afrough [5], a magnetic resonance method is applied to investigate the diffusion of liquids in geological porous materials, showing that the analysis can be more complicated than commonly believed. In the paper by Nathalie Bergeon, Guillaume Reinhart, Fatima L. Mota, Nathalie MangelinckNoël and Henri Nguyen-Thi [6], microgravity experiments are compared to ground-based ones in order to remove buoyancy forces and detect only the diffusive effects that lead to the solidification processes of metal alloys, comparing experimental results to existing theories and numerical results. In the paper by Fabio Giavazzi, Antara Pal and Roberto Cerbino [7], a method is described to simultaneously investigate the rotational and translational diffusive behaviour of colloidal particles by means of a simple microscope and a modification of the well-known differential dynamic microscopy method [8].

\subsection{Convection}

Convection is a macroscopic transport mechanism that is ubiquitous in nature. Convection is often driven by the force of gravity and initiated by the amplification of non-equilibrium fluctuations, as recently shown in the literature [9]. In the paper by Bingchuan Nie, Yutao Shao and Feng $\mathrm{Xu}[10]$, the authors explore the impact of boundary conditions when isothermal and isoflux conditions coexist in different parts of the section of a wall, with possible applications to the study of build- 
ing envelopes and their thermal performance. In the paper by Noureddine Latrache and Innocent Mutabazi [11], the transition to turbulence is investigated in Taylor-Couette flow of a viscoelastic polymer solution by varying the rotation speed of the inner cylinder of the apparatus. A similar apparatus is utilised in the paper by Awasthi and Hoshoudy [12], where the impact of heat and mass transfer on instability are investigated. Finally, in the paper by Thameem Basha and Sivaraj [13], the flow of blood containing nanoparticles through a porous tube is investigated for possible medical applications.

Acknowledgements We are pleased to thank all the authors and reviewers for their contribution and positive reply to our editorial request and for submitting contributions of scientific relevance. We also acknowledge the great efficiency and pleasant collaboration from the entire journal staff, as well as their competence and patience.

\section{References}

1. M. Ye, L. Zino, Ž Mlakar, J.W. Bolderdijk, H. Risselada, B.M. Fennis, M. Cao, Collective patterns of social diffusion are shaped by individual inertia and trendseeking. Nat. Commun. 12, 5698 (2021). https://doi. org/10.1038/s41467-021-25953-1

2. F. Croccolo, H.E. Roman, Spreading of infections on random graphs: a percolation-type model for COVID19. Chaos Solitons Fractals 139, 110077 (2020). https:// doi.org/10.1016/j.chaos.2020.110077

3. B. Afsharizand, P.H. Chaghoei, A.A. Kordbacheh, A. Trufanov, G. Jafari, Market of stocks during crisis looks like a flock of birds. Entropy 22, 1038 (2020). https:// doi.org/10.3390/e22091038

4. F. Croccolo, J.M. Ortiz de Zárate, J.V. Sengers, Nonlocal fluctuation phenomena in liquids. Eur. Phys. J. E 39, 125 (2016). https://doi.org/10.1140/epje/ i2016-16125-3

5. A. Afrough, Magnetic resonance free induction decay in geological porous materials. Eur. Phys. J. E 44, 107 (2021). https://doi.org/10.1140/epje/ s10189-021-00110-0
6. N. Bergeon, G. Reinhart, F.L. Mota, N. MangelinckNoël, H. Nguyen-Thi, Analysis of gravity effects during binary alloy directional solidification by comparison of microgravity and Earth experiments with in situ observation. Eur. Phys. J. E 44, 98 (2021). https://doi.org/ 10.1140/epje/s10189-021-00102-0

7. F. Giavazzi, A. Pal, R. Cerbino, Probing rototranslational diffusion of small anisotropic colloidal particles with a bright-field microscope. Eur. Phys. J. E 44, 61 (2021). https://doi.org/10.1140/epje/ s10189-021-00063-4

8. R. Cerbino, V. Trappe, Differential dynamic microscopy: probing wave vector dependent dynamics with a microscope. Phys. Rev. Lett. 100, 188102 (2008). https://doi.org/10.1103/PhysRevLett.100.188102

9. F. Giavazzi, A. Vailati, Scaling of the spatial power spectrum of excitations at the onset of solutal convection in a nanofluid far from equilibrium. Phys. Rev. E 80, 015303(R) (2009). https://doi.org/10.1103/PhysRevE. 80.015303

10. B. Nie, Y. Shao, F. Xu, An experimental study of natural convection on a vertical surface with neighboring isothermal and isoflux heating. Eur. Phys. J. E 44, 104 (2021). https://doi.org/10.1140/epje/ s10189-021-00105-x

11. N. Latrache, I. Mutabazi, Transition to turbulence via flame patterns in viscoelastic Taylor-Couette flow. Eur. Phys. J. E 44, 63 (2021). https://doi.org/10.1140/epje/ s10189-021-00067-0

12. M.K. Awasthi, G.A. Hoshoudy, Study of heat and mass transport on the instability of a swirling viscoelastic liquid film. Eur. Phys. J. E 44, 36 (2021). https://doi.org/ 10.1140/epje/s10189-021-00048-3

13. H. Thameem Basha, R. Sivaraj, Exploring the heat transfer and entropy generation of $\mathrm{Ag} / \mathrm{Fe} 3 \mathrm{O} 4-\mathrm{blood}$ nanofluid flow in a porous tube: a collocation solution. Eur. Phys. J. E 44, 31 (2021). https://doi.org/10.1140/ epje/s10189-021-00024-x 